\title{
La comunicación interna como desafío en las PyMES comerciales de Guayaquil
}

\section{Internal communication as a challenge in the commercial PySMES of Guayaquil}

Ana María Salazar Merchán

Universidad Ecotec, Ecuador

Autor para correspondencia: ansalazar@ecotec.edu.ec

Fecha de recepción: 24 de Mayo de 2017 - Fecha de aceptación: 15 de Septiembre de 2017

Resumen: El artículo fundamenta la comunicación interna como una herramienta eficaz para el buen manejo de clima laboral en todas las organizaciones. Mediante observación participante, la autora detecta una debilidad de este factor tan importante para la gestión empresarial, específicamente en las pymes del sector comercial de la ciudad de Guayaquil. Las pymes en Ecuador, siendo numerosas tienen un cometido muy desafiante y a su vez, la ventaja de la flexibilidad que las caracteriza, especialmente el poder adaptarse a las nuevas tecnologías, la inclusión de la mano de obra, y la respuesta positiva a los cambios de mercado. La comunicación es en esencia una herramienta estratégica para los procesos de redefinición de las relaciones de la empresa con sus públicos. Con los aportes de la comunicación, la cultura y la identidad corporativa se pueden intensificar como una sola acción empresarial ya que estos aspectos componen el sistema nervioso central de todos los procesos de la dinámica integral de la organización. Estas prácticas no son únicamente aplicables para grandes empresas o corporaciones internacionales, el crecimiento y sostenibilidad de las instituciones dependen también del buen gobierno corporativo; es una necesidad imperiosa a nivel macro, si consideramos el rol importante que tienen estas empresas en la estructura del país.

Palabras Clave: pymes; comunicación interna; clima laboral; cultura organizacional

Abstract: The article bases internal communication as an effective tool for the good management of work climate in all organizations. Through participant observation, the author detects a weakness of this factor so important for business management, specifically in SMEs in the commercial sector of the city of Guayaquil. PyMES in Ecuador have a very challenging role and, in turn, the advantage of the flexibility that characterizes them, especially being able to adapt to new technologies, the inclusion of labor, and the positive response to changes in market. Communication is essentially a strategic tool for the processes of redefinition of the relations of the company with its public. With the contributions of communication, culture and corporate identity can be intensified as a single business action as these aspects make up the central nervous system of all processes of the integral dynamics of the organization. These practices are not only applicable to large corporations or international corporations, the growth and sustainability of institutions also depend on good corporate governance; Is an imperative need at the macro level, if we consider the important role that these companies have in the structure of the country.

Key words: PyMES; internal communication; work climate; organizational culture 


\section{Introducción}

La comunicación ha evolucionado tanto, que se abren nuevas oportunidades para consolidar nuevas disciplinas. La clave del éxito de una política de comunicación interna reside en tener una buena planificación estratégica. Joan Costa, importante referente en el campo teórico del diseño y la comunicación, con numerosas publicaciones, menciona:

"La Dirección de Comunicación es una dirección central única. Lleva a cabo una visión y una responsabilidad de conjunto sobre las comunicaciones en y de la empresa, coordinadas con la estrategia general y los objetivos del negocio. Tiene una misión estratégica en el plano comunicacional/relacional dentro de la estrategia general de la empresa y en los asuntos importantes. Orienta y supervisa las acciones institucionales y de comunicación de las diferentes Direcciones Generales o Vicepresidencias. Y tiene una responsabilidad directa sobre la imagen corporativa" (Costa, 2004, p. 90).

Los vínculos más fuertes que unen al trabajador con su empresa son por adhesión e identificación con los objetivos del trabajo. Es primordial trabajar en los valores buscando solidez en la cultura y la comprensión de cómo la labor de cada uno contribuye en beneficio de la organización. Al respecto Lillian J. LeBlanc (2014) sostiene que:

"Crear un gran lugar para trabajar es fácil cuando usted tiene una gran cultura pues es probable que los colaboradores estén más comprometidos y felices en organizaciones donde la confianza abunda y la comunicación fluye libremente."

"La comunicación interna es una herramienta de gestión que también puede entenderse como una técnica" (Brandolini, González Frigoli, Hopkins, 2009, p. 24); estos autores afirman que el comunicador debe generar estrategias integrales que ayuden a gestionar lo más íntimo de la empresa. El interior de las organizaciones requiere de la gestión de un especialista en comunicación para plantear tácticas de comunicación externa e interna de forma integral, como una herramienta de valor estratégico:

“(...) ya que no sólo sirve para optimizar la gestión de los procesos, la satisfacción de los empleados y el mejor funcionamiento de los equipos, sino que logra que las personas, los trabajadores -verdadero eje de la compañía- se conviertan en verdaderos embajadores de la marca a la que representan, tanto cuando prestan un servicio en nombre de la compañía como cuando contribuyen a la proyección social de la imagen positiva de la compañía fuera del entorno laboral." (Losada, 2013, p. 12).

La comunicación interna existe en todas las organizaciones, ya sea de manera formal o informal, pero es importante que exista un conocimiento desde los altos mandos, que ofrezca soporte a este modelo de gestión de la comunicación, que dé paso al uso de nuevas tecnologías para una mayor difusión de los mensajes, generando así, un sentimiento de pertenencia a la empresa. Según la cultura que posea la organización, se conseguirá autonomía y empoderamiento para el logro de objetivos.

El flujo de comunicación también varía según su dirección: 
Tabla 1. Flujo de la comunicación

\begin{tabular}{ll}
\hline DIRECCIÓN & \multicolumn{1}{c}{ FLUJO } \\
\hline Ascendente & $\begin{array}{l}\text { Envío de mensajes desde los subordinados a los superiores } \\
\text { continuando su ascenso por la cadena jerárquica. }\end{array}$ \\
Descendente & $\begin{array}{l}\text { Envío de mensajes de los superiores a los subordinados. } \\
\text { Uno de los propósitos principales es proporcionar } \\
\text { instrucciones específicas de trabajo. } \\
\text { La comunicación horizontal es básicamente la que se } \\
\text { genera entre los miembros de un mismo nivel jerárquico. }\end{array}$ \\
& $\begin{array}{l}\text { Comunicación que se genera entre las } \\
\text { diferentes posiciones de la jerarquía. }\end{array}$ \\
\hline
\end{tabular}

En la comunicación interna es necesario tener en cuenta las diversas dimensiones que constituyen su espacio de socialización y retroalimentación, así como la diferencia en los flujos de mensajes existentes ante los distintos niveles de las estructuras organizacionales, descendentes y ascendentes, horizontales o transversales. De la misma forma, es indispensable diferenciar el tono de la comunicación a través del estudio de los modos y áreas en que esta fluye (Villafañe, 1993).

Elias y Mascaray (2003, p. 37), indican “La empresa necesita, cada día más, la aportación de la iniciativa y creatividad de la totalidad de sus elementos integrantes, sean dos, doscientos o veinte mil". Al planificar la comunicación interna como se gestionan la mayoría de los procesos, se logra desarrollar y aplicar con éxito los objetivos de la empresa; y, si no se la gestiona correctamente, pueden generarse los conocidos rumores. Según Robbins (1996), los rumores dificultan el normal desarrollo de las actividades disminuyendo la capacidad de las relaciones interpersonales. Además, generan un clima laboral no adecuado, dificultando los procesos internos de la compañía. Schneider y Reichers (1983) mencionan en sus estudios que las personas generan una percepción general sobre la organización, que sirve como mapa cognitivo del funcionamiento de la organización, así se determina un comportamiento adecuado ante cada situación.

Tabla 2. Características y tendencias sobre Clima Laboral, autores destacados.

\begin{tabular}{lll}
\hline Autores & Características & Tendencia \\
\hline & Características & Percepción Que Tiene Los Miembros De La \\
\multirow{3}{*}{ Glick, } & Estructurales: & Organización Acerca De: Reglas, Procedimientos, \\
1985 & Confianza En Liderazgo, & Diligencias Y Limitaciones Enfrentados En Su \\
& Flujo De Comunicación, & Desempeño (Variables Constantes Dentro De La \\
& Calidad En Servicio. & Organización Y Definidas Por La Dirección De La \\
\hline
\end{tabular}

Fuente: Elaboración del autor

Clima laboral y cultura organizacional se relacionan con atributos propios del comportamiento, percepciones, valores y actitudes; poseen un carácter tan complejo y dinámico que en ocasiones suelen mezclarse sus características. La tabla a continuación, muestra las diferencias entre Cultura y Clima Organizacional. 
Tabla 3. Diferencias entre Cultura y Clima organizacional

\begin{tabular}{lll}
\hline \multicolumn{1}{c}{ Diferencias } & Cultura Organizacional & Clima Organizacional \\
\hline $\begin{array}{l}\text { Epistemología } \\
\text { Punto de vista }\end{array}$ & $\begin{array}{l}\text { Contextualización e Ideografía } \\
\text { Étnico (nativo) } \\
\text { Observación de campo, estudio } \\
\text { cuantitativo }\end{array}$ & $\begin{array}{l}\text { Comparativa y Homotética } \\
\text { Ético (Investigador) }\end{array}$ \\
Nivel de análisis & Valores y presunciones subyacentes & Estudio cualitativo \\
$\begin{array}{l}\text { Orientación en } \\
\text { tiempo }\end{array}$ & Evolución histórica & $\begin{array}{l}\text { Consenso sobre } \\
\text { percepciones }\end{array}$ \\
Fundaciones & Construcción social & \begin{tabular}{l} 
Instantánea, no histórica \\
\hline
\end{tabular}
\end{tabular}

Fuente: Adaptado de (Denison, 1996, pp. 619 - 654).

Para Martínez (2016):

En las organizaciones el clima laboral, tiene una importante influencia en las actitudes y conductas, así como es el desempeño empresarial reflexiona sobre la mejora del clima y la forma de intervenir en el cambio, mejorando el desempeño y los resultados de la organización (p. 44).

El trabajador, por encima de las materias primas y el capital, se ha convertido en el principal factor de producción. Como señala Robles:

"la integración y motivación del denominado trabajador del conocimiento se ha convertido en una verdadera prioridad para las grandes empresas, conscientes de que la inteligencia y el talento personal de sus empleados es la única materia prima que puede diferenciarlas de la competencia" (2001, p. 9).

Kaplan y Norton (1997, p. 143) indican "Los empleados satisfechos son una condición previa para el aumento de la productividad, de la rapidez de reacción, la calidad y el servicio al cliente."

Una de las definiciones más completas de comunicación interna es ofrecida por Horacio Andrade (1991, p. 32) donde la nombra como:

“el conjunto de actividades efectuadas por cualquier organización para la creación y mantenimiento de buenas relaciones con y entre sus miembros, a través del uso de diferentes medios de comunicación, que los mantengan informados, integrados y motivados para contribuir con su trabajo al logro de los objetivos organizacionales".

El gran desafío de la comunicación interna del siglo XXI es cuidar al cliente interno para mantenerlo satisfecho; solo así se podrá dar atención al cliente externo brindándole pronta solución a sus problemas y satisfaciendo sus necesidades (Muñiz, 2017).

\section{La investigación}


Según La Superintendencia de Compañías, organismo técnico con autonomía administrativa y económica, que vigila y controla la organización, actividades, funcionamiento, disolución y liquidación de las compañías y otras entidades en las circunstancias y condiciones establecidas por la Ley, existen 28.616 MIPYMES activas (micro, pequeñas y medianas compañías), registradas en la provincia del Guayas hasta el 3 de marzo del 2016. En la rama de Comercio al por mayor y menor, se encuentran 7.281.

El tipo de muestra que se consideró para esta investigación es aleatoria, se trabajó con un nivel de confianza del $95 \%$ y un error típico del $5 \%$ que son los datos estándares y generalmente aceptados para estos trabajos, cuya fórmula es la que se presenta a continuación:

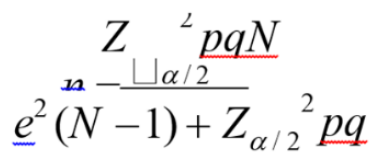

Dónde:

\section{N: Población}

n: muestra

$\alpha / 2$ : Nivel de confianza

$\mathrm{Z} \alpha / 2$ : Constante que depende del nivel de confianza

p y q: probabilidad de ocurrencia; "p": individuos que si poseen la característica de estudio, "q": individuos que no poseen la característica de estudio.

e: error típico

Tras la aplicación de la fórmula con un nivel de confianza de 95 y un margen de error del $5 \%$, se obtuvo que las encuestas debieran ser aplicadas a 379 empresas de la ciudad de Guayaquil.

De las preguntas correspondientes a la entrevista elaborada, la autora destaca: 


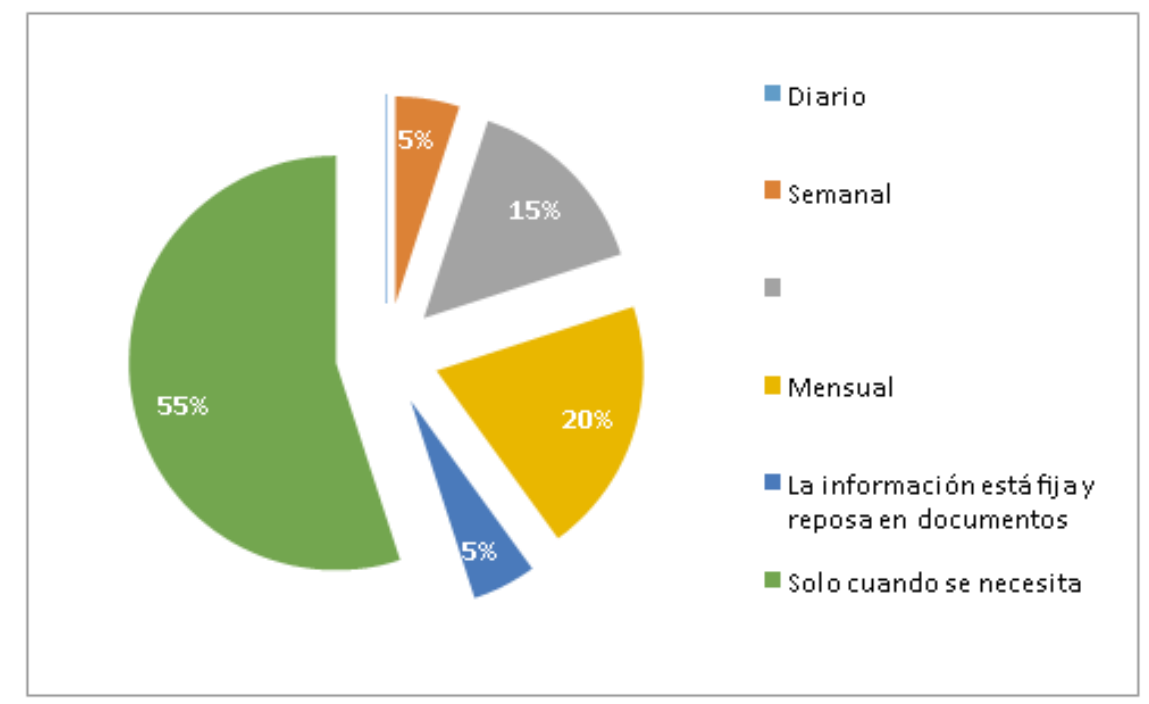

Figura 1. Pregunta: ¿Con qué frecuencia se envían comunicados a los colaboradores? Fuente: Elaboración del autor

El 55\% de las empresas encuestadas, reflejan que se realiza una comunicación interna sólo cuando es necesaria, no sigue una planificación estratégica, perdiendo la oportunidad de diseñar estrategias coherentes con la manera de ser y hacer de la empresa y su proyección externa e interna. La comunicación interna resulta fundamental en la estrategia global de la compañía ya que facilita el conocimiento de la actividad, de la información y de la cultura empresarial, crea vínculos comunes entre los colaboradores y la compañía, convierte a los trabajadores en los primeros embajadores de la marca, defendiendo su imagen positiva.

Al planificar la comunicación interna como se gestionan la mayoría de los procesos, se logrará desarrollar y aplicar con éxito los objetivos anuales traducidos en acciones concretas. Es importante establecer unos soportes de comunicación útiles, eficaces, rápidos y accesibles a todos.

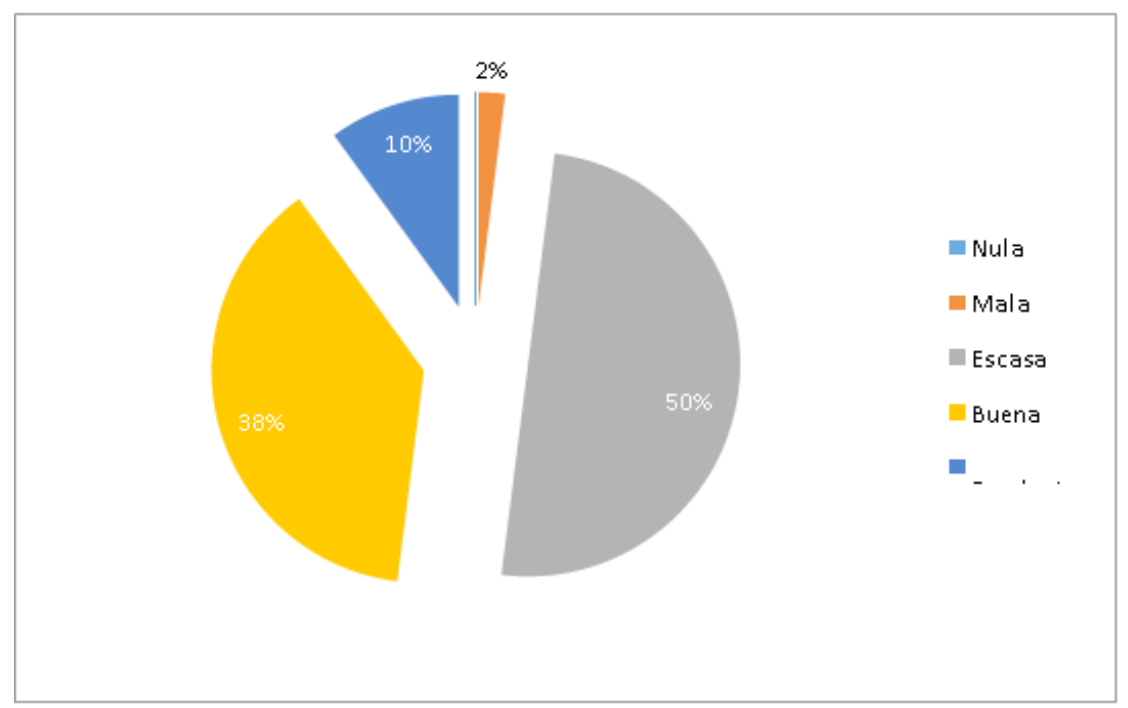


Figura 2. Indicación: Por favor, valore la comunicación interna en su empresa Fuente: Elaboración del autor

Confirmando el cuestionamiento anterior, la mitad de las organizaciones encuestadas, reflejan que la comunicación es escasa, que no existe una planificación que sustente el buen manejo de un valor clave en la organización. Las organizaciones son sistemas sociales abiertos que deben procesar información, hoy en día estamos viviendo una sociedad tecnológica debido a los distintos cambios que se han producido en la manera como se da la comunicación. Las TIC, Tecnologías de la información, ofrecen una serie de nuevas herramientas que se pueden utilizar de manera efectiva en muchos procesos: apoyando la toma de decisiones, funciones ejecutivas, la planificación, el trabajo en grupo, y por supuesto en la comunicación.

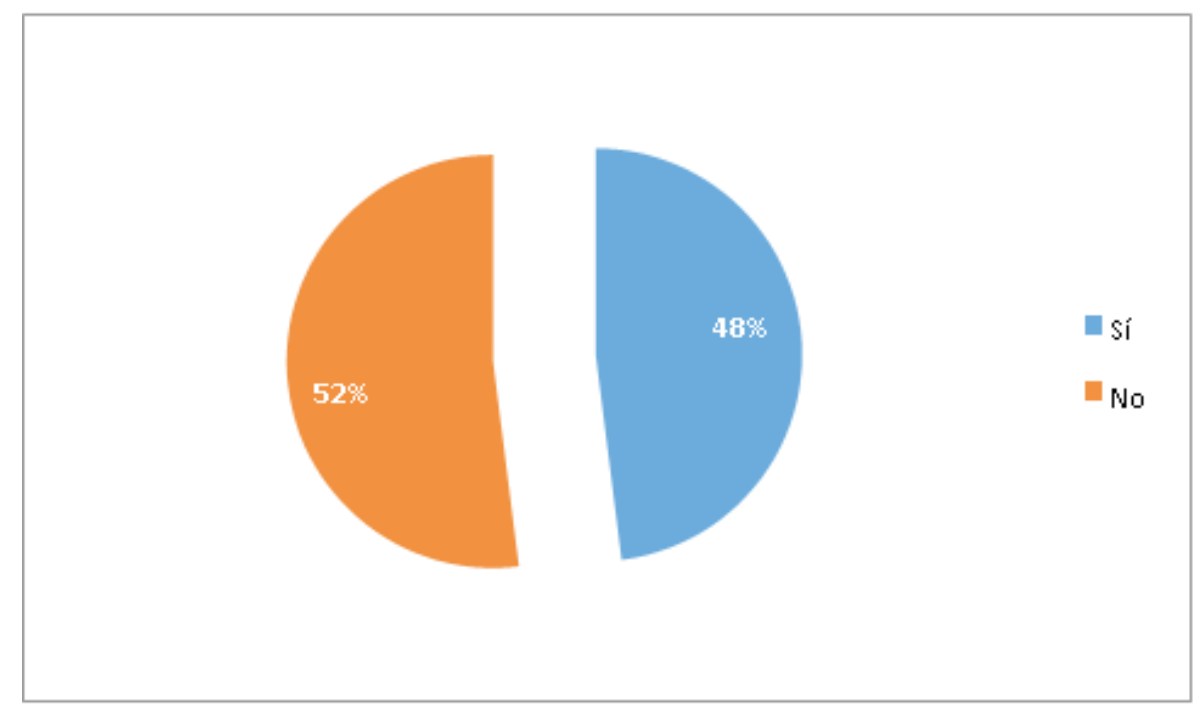

Figura 3. Pregunta: ¿Existe un departamento o un responsable para comunicación interna? Fuente: Elaboración del autor

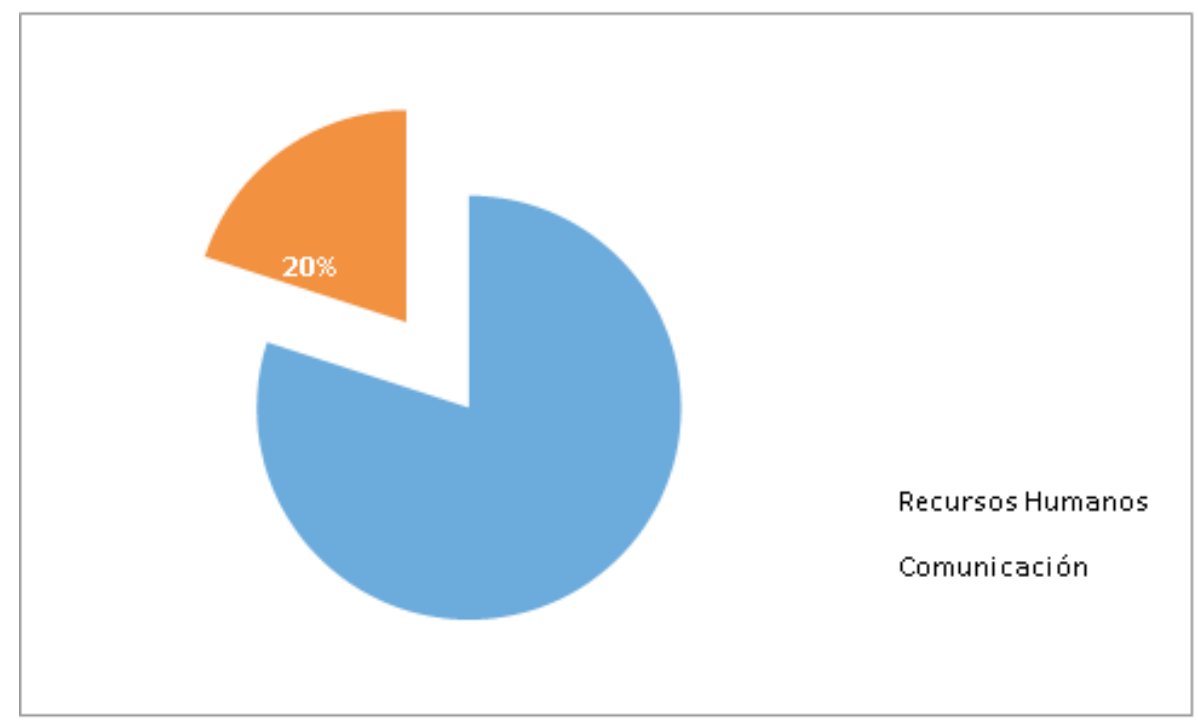

Figura 4. Pregunta: ¿La persona encargada tiene formación en RRHH o en Comunicación? 
Fuente: Elaboración del autor

Finalmente, y no menos importante, se quiso indagar si la persona responsable de la comunicación tenía formación en el área de Recursos Humanos o era un profesional en Comunicación. El 80\% del público escrutado, tiene formación en el área de Recursos Humanos, olvidando que este cargo representa el desarrollo de un nuevo perfil profesional y no un simple cambio de denominación, es un nuevo profesional para una nueva realidad como el más alto responsable de la Comunicación y la Cultura Organizacional.

Una de las tareas esenciales para el responsable de Comunicación es transmitir y ayudar a evolucionar la cultura corporativa, lo cual de alguna manera incluye a otras tareas, ya que si se logra establecer una cultura de servicio al cliente, incluyendo políticas y estrategias de comunicación, donde los colaboradores den siempre lo mejor, este hecho ayudará a transmitir la esencia de la corporación.

\section{Conclusión}

Para lograr una conexión con la comunicación interna es importante plantear una estrategia desde un punto de vista más humano, sin perder su funcionalidad, para así poder involucrar al personal de una manera más emocional, que incluya la participación dentro de toda la estructura organizacional. Usando valores como motivación y orgullo, y sobre todo escuchando a los públicos, se puede lograr un mejor manejo de comunicación interna.

La comunicación vista como un todo debe ser tratada de forma institucional, mercadológica y organizacional. Por ser portavoz de la institución, el responsable de Comunicación debe controlar puntualmente la información interna de la compañía; al establecer bien estos parámetros, el resto de la cultura organizacional en su mayor parte funcionaría adecuadamente.

La comunicación interna, nace bajo la necesidad de motivar al equipo humano y mantener grupos de trabajo competitivos además de inculcar una verdadera cultura corporativa, identificarlos y fidelizarlos a la organización.

Para enumerar las ventajas que la comunicación interna aporta a las empresas:

1. Transmitir la identidad corporativa a los colaboradores.

2. Promover un flujo de comunicación bidireccional: genera clima de confianza, mantener las buenas relaciones.

3. Mejora la productividad de la empresa al facilitar el intercambio de información vital.

4. Se está al tanto de los objetivos de la organización ayudando a su cumplimiento.

5. Facilita la adaptación a cambios dentro de la organización por consecuencia de crisis internas o externas.

6. Ayuda a motivar a los colaboradores, que influye en su nivel de productividad.

No es exclusiva de las grandes empresas, todas las organizaciones deben desarrollar adecuadas políticas de comunicación interna que contribuyan a implantar los cambios y a lograr los objetivos corporativos y estratégicos en la compañía. 
Un ejemplo muy sencillo de realizar para empezar tareas de comunicación, es crear un dossier o boletín de forma periódica -quizás mensual- que reúna información de medios de comunicación para mostrarla a los públicos, adicionalmente información con opinión de los consumidores con resultados de sondeo de opinión pública.

\section{Bibliografía}

Andrade, H. (1991). La Comunicación en las Organizaciones. México, México: Trillas.

Brandolini, A., González Frigoli, M. y Hopkins N. (2009). Comunicación interna: claves para una gestión exitosa. Buenos Aires: La Crujía.

Costa, J (2000). Comunicación empresarial: Nuevas tendencias en comunicación para potenciar la estrategia empresarial. España, Barcelona.

Costa, J. (2004). DirCom on-line. La Paz, Bolivia: Desing.

Denison, D. (julio, 1996). What is the difference between organizational culture and organizational climate? A native's point in the view on a decade of paradigm wars. Academy of Management Review, Vol. 21 No. 3, 619 - 654.

Elías, J. y Mascaray, J. (2003). Más allá de la Comunicación Interna: La Intracomunicación. Barcelona, España: Gestión 2000.

Kaplan, R.S., Norton, D.P. (1997). El Cuadro de Mando Integral: The Balance Score Card. Barcelona, España: Gestión 2000.

LeBlanc, L. (2014). Tres pasos para transformar su cultura. Recuperado en julio de 2014 de http://www.greatplacetowork.com.ec/publicaciones-y-eventos/blogs-y-noticias/707trespasos-para-transformar-su-cultura

Losada, J. C. (2013). Material teórico Cultura Organizacional y Comunicación Interna. Quito, Ecuador: UDLA

Martínez, M. J. (2016). Gestión Estratégica del Clima Laboral. Madrid: Editorial UNED.

Muñiz, R. (2017). Marketing XXI. Recuperado el 19 de 06 de 2017, de Marketing XXI: http://www.marketing-xxi.com/la-comunicacion-interna-119.htm

Robbins, S. (1996). Comportamiento Organizacional. Teoría y Práctica (7a. ed.). México, México: Prentice - Hall Hispanoamericana. S.A.

Robles, M. (2001). La comunicación interna en las entidades financieras andaluzas. El ejemplo de El Monte y CajaSur (tesina). Sevilla, España: Facultad de Comunicación. 
Schneider, B. y Reichers, A.E. (1983). On the etiology of climates. Personnel Psychology, Vol 36, p. 19-39

Villafañe, J. (1993). Imagen Positiva. Gestión Estratégica de la Imagen de la Empresa. Madrid: Pirámide. 